\title{
Stress concentration mitigation in clamped steel plates
}

\author{
Kanak Kalita \\ Dept. of Aerospace Engineering \& Applied Mechanics, IIEST, Shibpur Howrah, WB \\ E-mail: kanakkalita02@gmail.com
}

Copyright $\odot 2014$ Kanak Kalita. This is an open access article distributed under the Creative Commons Attribution License, which permits unrestricted use, distribution, and reproduction in any medium, provided the original work is properly cited.

\begin{abstract}
Steel is used widely in the construction of roads, railways, other infrastructure, appliances, and buildings. Most large modern structures, such as stadiums, skyscrapers, bridges, airports, are supported by a steel skeleton. Steel plates with internal cutouts are widely used in structural members. These cutouts are made into plates to meet various functional and structural requirements. However these cutouts create stress concentration and eventually reduce the mechanical strength of the structure. The present study aims at reducing this stress concentration around the central hole by introduction of a proposed scheme of auxiliary holes. Reduction in stress concentration with symmetric and asymmetric auxiliary holes is studied. Findings of the study are made available here in both numerical data and graphical form.
\end{abstract}

Keywords: Auxiliary Hole, Cutout Plate, Finite Element Method, Stress Mitigation, Stress Analysis.

\section{Introduction}

It is a well-known fact that the presence of discontinuity in a stressed member creates highly localized stresses at the vicinity of the cutout. In recent years, researchers have put enormous amount of effort in investigating techniques for analysis and mitigation of stress concentration. The failure of structures due to stress concentration at any discontinuity has been baffling engineers for long. It has been found that structure failures in ships, offshore structures, boilers or high rise buildings subjected to natural calamities is due to stress concentration. Stress concentration mainly occurs due to discontinuities in continuum. Due to stress concentration the magnitude of the maximum stress occurring in any discontinuity is comparatively higher than the nominal stress. Stress concentration cause strength degradation and premature failure of structures because of fatigue cracking and plastic deformation frequently occurring at these points. Motok [1] carried out stress concentration studies on the contour of a plate opening of an arbitrary corner radius of curvature. Heywood [2] reported that stress concentration can be reduced by introducing smaller auxiliary holes on either side of the original hole, which smoothen the flow of the tensile principal stress trajectories past the original hole. Rajaiah et al. [3] proposed hole shape optimization for stress mitigation in a finite plate by photo elasticity method. They introduced auxiliary holes around central hole for mitigation of SCF and also optimized the shape of circular holes. Meguid [4] presented a technique for reduction of SCF in a uni-axially loaded plate with two coaxial holes by introducing defense hole system- material removal in the form of circular holes. Defense hole system is a technique of material removal for stress mitigation. Finite element method was used for analysis. A comprehensive plane stress finite element study of the effect of material removal upon mitigation of elastic SCF in a uni-axially loaded plate with two coaxial holes was made. Reduction in maximum SCF ranging from 7.5\% to $11 \%$ could be achieved. Giare et al. [5] presented a method for the reduction of stress concentration in an isotropic plate by using composite material rings around the hole. They have reported the reduction in stress concentration factor by reinforcement. Kalita et al. [6] has studied the variation of deflection and induced stresses due to presence of central cutouts under transverse loading. They have used small auxiliary holes around the central square hole to mitigate stresses in orthotropic and isotropic plates [7], [8]. 


\section{Equations used}

Stress analysis of an elastic body is usually three dimensional problems. But, most of the practical problems appear in the state of plane stress or plane strain. Stress analysis of three-dimensional bodies under plane stress or plane strain can be treated as two- dimensional problems. The solution of two-dimensional problems requires the integration of the different equations of equilibrium together with the compatibility equations and boundary conditions. If body force is neglected, the equations to be satisfied are:

$\frac{\partial \sigma_{x}}{\partial x}+\frac{\partial \sigma_{x y}}{\partial y}=0$

$\frac{\partial \sigma_{y}}{\partial y}+\frac{\partial \sigma_{x y}}{\partial x}=0$

$\left(\frac{\partial^{2}}{\partial x^{2}}+\frac{\partial^{2}}{\partial y^{2}}\right)\left(\sigma_{x}+\sigma_{y}\right)=0$

Substitution of stress components by displacement components u and v into Eq. (1) to (3) makes Eq. (3) redundant and Eq. (1) and (2) transforms to

$\frac{\partial^{2} u}{\partial x^{2}}+\frac{(1-v)}{2}\left(\frac{\partial^{2} u}{\partial y^{2}}\right)+\frac{(1+v)}{2}\left(\frac{\partial^{2} v}{\partial x \partial y}\right)=0$

$\frac{\partial^{2} v}{\partial y^{2}}+\frac{(1-v)}{2}\left(\frac{\partial^{2} v}{\partial x^{2}}\right)+\frac{(1+v)}{2}\left(\frac{\partial^{2} u}{\partial x \partial y}\right)=0$

Now we need to find $u$ and $v$ from a two dimensional field satisfying the two partial differential Eq. (4) and (5).Instead of determining the two functions $\mathrm{u}$ and $\mathrm{v}$ the problem can be reduced to solving a single function $\psi(\mathrm{x}, \mathrm{y})$, which can be determined by satisfying Eq. (4) and (5). The displacement potential function $\psi(\mathrm{x}, \mathrm{y})$ can be defined as

$u=\frac{\partial^{2} \psi}{\partial x \partial y}$

$v=-\left[(1-v)\left(\frac{\partial^{2} \psi}{\partial y^{2}}\right)+2\left(\frac{\partial^{2} \psi}{\partial x^{2}}\right)\right] /(1-v)$

By the above definitions the displacement components $u$ and $v$ satisfies Eq. (4) and the only condition reduced from Eq.

(5) that the function $\psi(\mathrm{x}, \mathrm{y})$ has to satisfy is

$\frac{\partial^{4} \psi}{\partial x^{4}}+2\left(\frac{\partial^{4} \psi}{\partial x^{2} \partial y^{2}}\right)+\frac{\partial^{4} \psi}{\partial y^{4}}=0$

So, now the problem is to evaluate a single function $\Psi(\mathrm{x}, \mathrm{y})$ from the bi-harmonic Eq. (7), satisfying the boundary conditions specified at the boundary [9].

\section{Finite element formulation}

An all side clamped rectangular steel plate of $1500 \mathrm{~mm}$ x $1000 \mathrm{~mm}$ x $2 \mathrm{~mm}$ (A x B x t) with a central square cutout of side (b) $200 \mathrm{~mm}$ is considered for study. Material properties of the plate are taken as $\mathrm{E}=2 \times 10^{11} \mathrm{~N} / \mathrm{m}^{2}$ and Poisson's ratio 0.3. A uniformly distributed load of $1 \mathrm{~N}$ is applied as transverse load. An 8 node shell element (Fig. 1) with element length of $1 \mathrm{~mm}$ near discontinuity and about $2 \mathrm{~mm}$ at places away from the central and auxiliary hole is used throughout the study. The element has eight nodes with six degrees of freedom at each node: translations in the $\mathrm{x}$, $\mathrm{y}$, and $\mathrm{z}$ axes, and rotations about the $\mathrm{x}, \mathrm{y}$, and $\mathrm{z}$-axes. Thus each element has 48 degree of freedom in total.

Fig. 2 illustrates the dimensions taken. All variation of distances of auxiliary holes from the periphery of the central cutout which are $X_{1}, X_{2}, Y_{1}$ and radius of the auxiliary holes which are $r_{1}, r_{2}$ are taken as functions of side of the central cutout (i.e. b). Distance of the auxiliary holes (i.e. $\mathrm{X}_{1}, \mathrm{X}_{2}, \mathrm{Y}_{1}$ ) is varied as distance/cutout side length ratio (i.e. $\mathrm{X}_{1} / \mathrm{b}$, $\mathrm{X}_{2} / \mathrm{b}, \mathrm{Y}_{1} / \mathrm{b}$ ). Four models of auxiliary hole placement are considered. One important concern while placing the auxiliary holes is to keep their sizes minimum.

Model 1: A single auxiliary hole with radius $r_{1}$ is placed to the left of central hole on y center line at distance $\mathrm{X}_{1}$ from the central hole. This model induces asymmetry to an otherwise symmetrical plate.

Model 2: Two auxiliary holes with radius $\mathrm{r}_{1}$ are placed on either side of the central hole on y center line at distance $\mathrm{X}_{1}$. Also the distance is kept $\mathrm{X}_{1}$ and radius $\mathrm{r}_{1}$ for both the holes to attain symmetry.

Model 3: Four auxiliary holes are placed, two same as model 2 and other two with radius $r_{1}$ at $x$ center line at distance $\mathrm{Y}_{1}$. Thus four symmetrical holes are placed all around the central hole.

Once an optimized location of $\mathrm{X}_{1}$ and $\mathrm{Y}_{1}$ and optimum radius $\mathrm{r}_{1}$ is obtained, we can carry out model 4 to check the feasibility of using a 2 nd pair of auxiliary holes. 


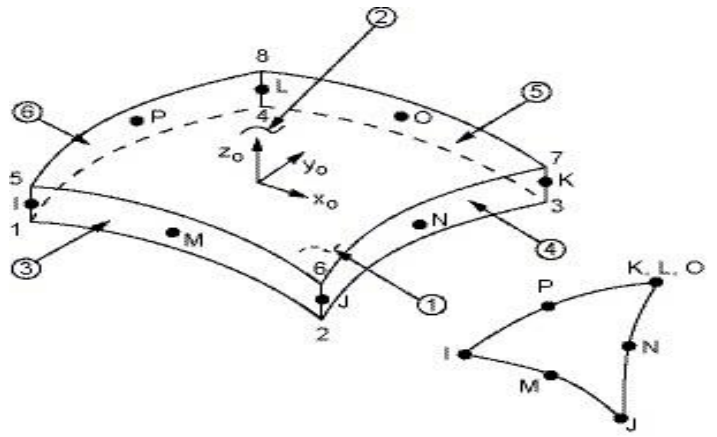

Fig. 1: 8 Node Shell Element used in the Analysis

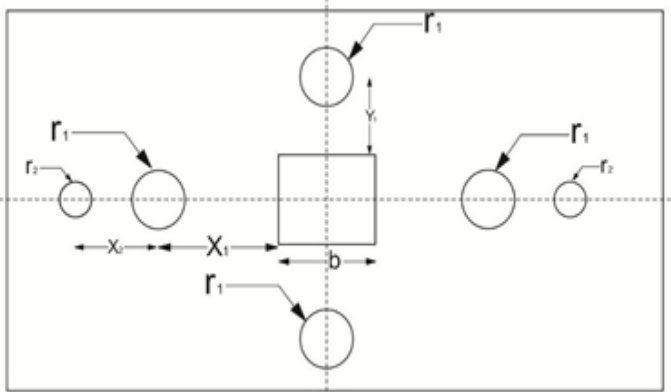

Fig. 2: Rectangular Plate with Central Cutout and Auxiliary Holes.

Model 4: Two more auxiliary holes with radius $r_{2}$ at a distance $X_{2}$ in the $y$ center line are placed around the arrangement obtained from model 3's optimization.

\section{Results and observation}

Fig. 3 shows the stress contour of rectangular plate with an internal square cutout. It is seen that the stress is maximum at the corners of the cutout and at the support edges. The maximum von Mises stress is $119638 \mathrm{~N} / \mathrm{m}^{2}$ whereas a maximum stress of $100619 \mathrm{~N} / \mathrm{m}^{2}$ is induced in a solid plate without any cutout under the same loading conditions. The percent reduction in Von Mises stress by using model 1 to model 4 are shown in graphical form in fig. 4, 6, 8, 10 and the stress contour plots are shown in fig 5, 7,9,11.

For $r_{1} / b=0.05$ it is seen that model 1 shows small reduction in stress at the proximity of central hole, but as we increase the distance from the central hole they become a source of stress raiser at $\mathrm{X}_{1} / \mathrm{b}=0.1$, further increase in $\mathrm{X}_{1} / \mathrm{b}$ to 1.5 causes small reduction in stress. If the $r_{1} / b$ ratio is increased to 0.1 it is seen that at proximity to the central hole the auxiliary holes become stress raiser but at sufficient distance of $X_{1} / b=1.5$ a small reduction in stresses is seen. It is also seen that the maximum stress reduction that can be obtained by model 1 is by a radius $r_{1} / b=0.2$ at $X_{1} / b=1.5$ which is about 5\%. Comparison of stress plot in Fig. 3 and Fig. 5 reveals clearly that the presence of an auxiliary hole on the left side of central hole distributes the stresses better and hence is lower in Fig. 5.

Table 1: Percent Reduction in Von Mises Stress for Model $1 \&$ Model 2.

\begin{tabular}{|c|c|c|c|c|c|c|}
\hline Model & $\mathrm{X}_{1}(\mathrm{~mm})$ & $\mathrm{r}_{1}(\mathrm{~mm})$ & $\mathrm{X}_{1} / \mathrm{b}$ & $\mathrm{r}_{1} / \mathrm{b}$ & Von Mises stress $\left(\mathrm{N} / \mathrm{mm}^{2}\right)$ & $\%$ reduction in stress \\
\hline \multirow{9}{*}{1} & 100 & \multirow{3}{*}{10} & 0.5 & \multirow{3}{*}{0.05} & 119441 & 0.165 \\
\hline & 200 & & 1 & & 124482 & -4.049 \\
\hline & 300 & & 1.5 & & 118914 & 0.605 \\
\hline & 100 & \multirow{3}{*}{20} & 0.5 & \multirow{3}{*}{0.1} & 122104 & -2.061 \\
\hline & 200 & & 1 & & 129866 & -8.549 \\
\hline & 300 & & 1.5 & & 118593 & 0.873 \\
\hline & 100 & \multirow{3}{*}{40} & 0.5 & \multirow{3}{*}{0.2} & 118600 & 0.868 \\
\hline & 200 & & 1 & & 118501 & 0.950 \\
\hline & 300 & & 1.5 & & 113692 & 4.970 \\
\hline \multirow{9}{*}{2} & 100 & \multirow{3}{*}{10} & 0.5 & \multirow{3}{*}{0.05} & 120124 & -0.406 \\
\hline & 200 & & 1 & & 119850 & -0.177 \\
\hline & 300 & & 1.5 & & 119057 & 0.486 \\
\hline & 100 & \multirow{3}{*}{20} & 0.5 & \multirow{3}{*}{0.1} & 119906 & -0.224 \\
\hline & 200 & & 1 & & 119383 & 0.213 \\
\hline & 300 & & 1.5 & & 119630 & 0.007 \\
\hline & 100 & \multirow{3}{*}{40} & 0.5 & \multirow{3}{*}{0.2} & 112453 & 6.006 \\
\hline & 200 & & 1 & & 124258 & -3.862 \\
\hline & 300 & & 1.5 & & 116755 & 2.410 \\
\hline
\end{tabular}




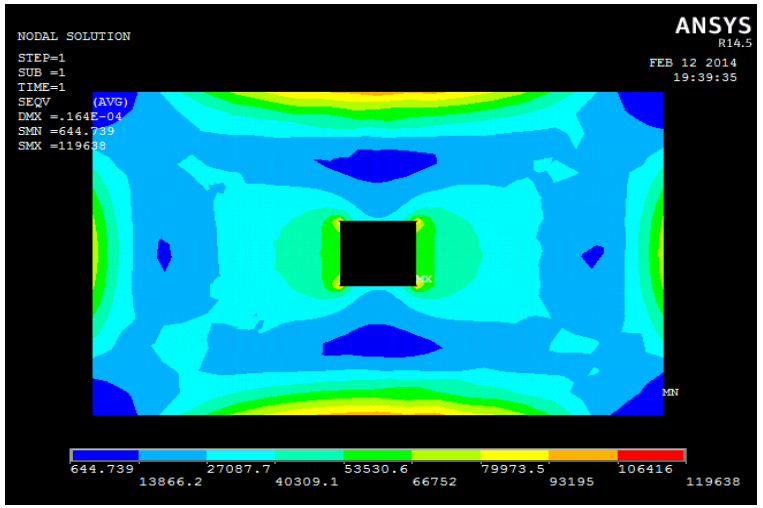

Fig. 3: Stress Contour Plot of Plate with Central Cutout

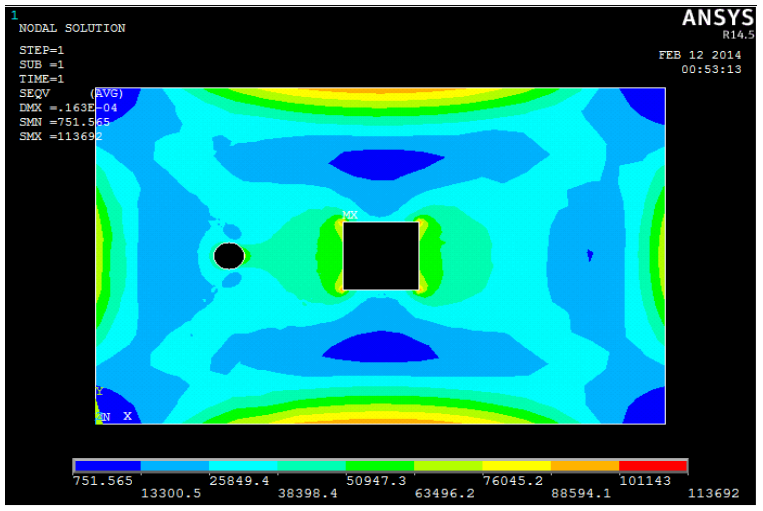

Fig. 5: Stress Contour Plot for Model 1 with $r_{1}=0.2 b, X_{1}=1.5 b$

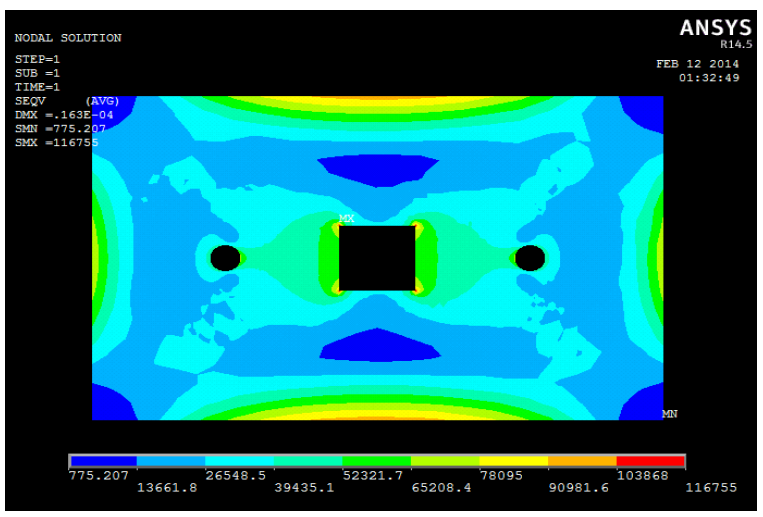

Fig. 7: Stress Contour Plot for Model 2 with $r_{1}=0.2 b, X_{1}=1.5 b$

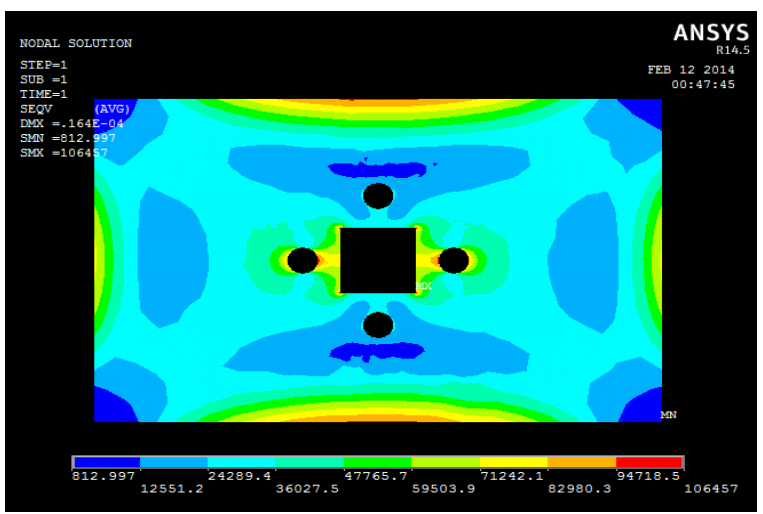

Fig. 9: Stress Contour Plot for Model 3 with $r_{1}=0.2 b, X_{1}=0.5 b$

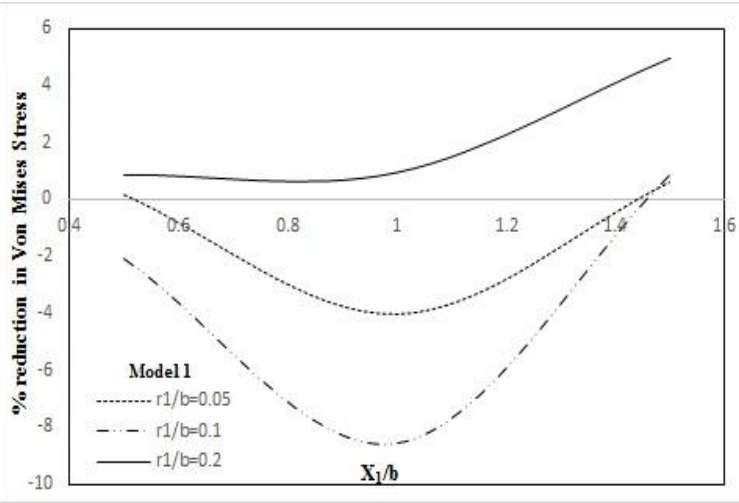

Fig. 4: Percent Reduction in Stress Vs. $X_{1} / b$ for Model 1

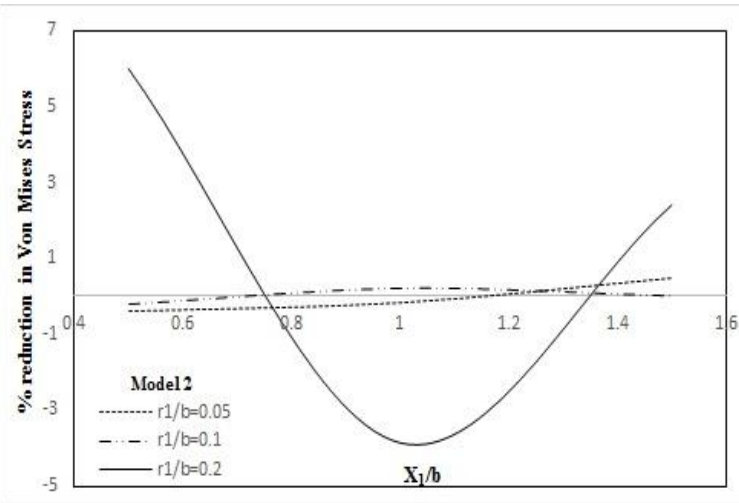

Fig. 6: Percent Reduction in Stress Vs. $\mathrm{X}_{1} / \mathrm{b}$ for Model 2

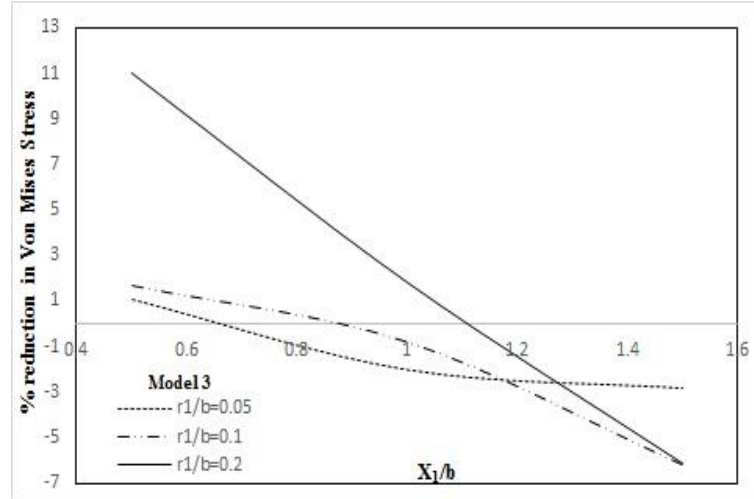

Fig. 8: Percent Reduction in Stress Vs. $X_{1} / b$ for Model 3

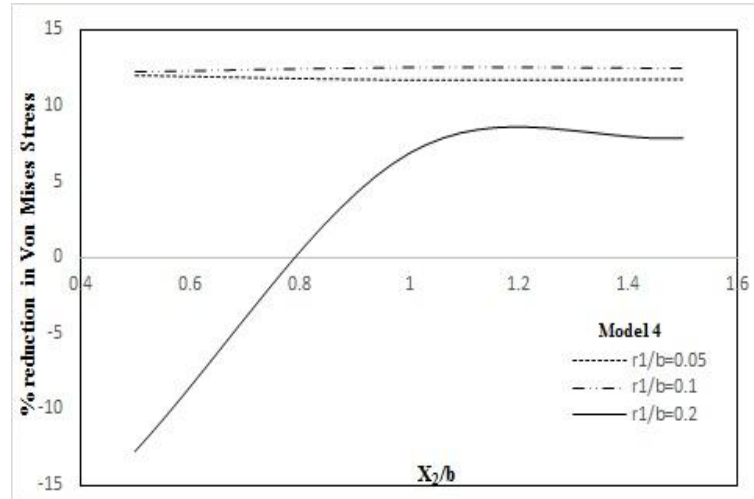

Fig. 10: Percent Reduction in Stress Vs. $\mathrm{X}_{2} / \mathrm{b}$ for Model 4 
Table 2: Percent Reduction in Von Mises Stress for Model 3

\begin{tabular}{|c|c|c|c|c|c|}
\hline $\mathrm{X}_{1}=\mathrm{Y}_{1}(\mathrm{~mm})$ & $\mathrm{r}_{1}(\mathrm{~mm})$ & $\mathrm{X}_{1} / \mathrm{b}$ & $\mathrm{r}_{1} / \mathrm{b}$ & Von Mises stress $\left(\mathrm{N} / \mathrm{mm}^{2}\right)$ & $\%$ reduction in stress \\
\hline 100 & & 0.5 & & 118334 & 1.090 \\
\hline 200 & 10 & 1 & 0.05 & 122023 & -1.994 \\
\hline 300 & & 1.5 & & 122995 & -2.806 \\
\hline 100 & & 0.5 & & 117624 & 1.683 \\
\hline 200 & 20 & 1 & 0.1 & 120604 & -0.807 \\
\hline 300 & & 1.5 & & 127092 & -6.230 \\
\hline 100 & & 0.5 & & 106457 & 11.017 \\
\hline 200 & 40 & 1 & 0.2 & 117461 & 1.820 \\
\hline 300 & & 1.5 & & 126989 & -6.144 \\
\hline
\end{tabular}

The best results obtained for model 2 is by using $r_{1} / b=0.2$ at a distance $X_{1} / b=0.5$ for which reduction in stresses is $6 \%$. At a distance $\mathrm{X}_{1} / \mathrm{b}=1.5$ about $2.5 \%$ reduction in stress is seen with $r_{1} / b=0.2$. Stress contour plot in Fig. 7 shows that the two auxiliary holes aid in distributing the region of high stress.

Table 3: Percent Reduction in Von Mises Stress for Model 4

\begin{tabular}{|c|c|c|c|c|c|}
\hline $\mathrm{X}_{2}(\mathrm{~mm})$ & $\mathrm{r}_{2}(\mathrm{~mm})$ & $\mathrm{X}_{2} / \mathrm{b}$ & $\mathrm{r}_{2} / \mathrm{b}$ & Von Mises stress $\left(\mathrm{N} / \mathrm{mm}^{2}\right)$ & $\%$ reduction in stress \\
\hline 100 & & 0.5 & & 105248 & 12.028 \\
\hline 200 & 10 & 1 & 0.05 & 105665 & 11.679 \\
\hline 300 & & 1.5 & & 105600 & 11.734 \\
\hline 100 & & 0.5 & & 105021 & 12.218 \\
\hline 200 & 20 & 1 & 0.1 & 104613 & 12.559 \\
\hline 300 & & 1.5 & & 104704 & 12.483 \\
\hline 100 & & 0.5 & & 134929 & -12.781 \\
\hline 200 & 40 & 1 & 0.2 & 111434 & 6.857 \\
\hline 300 & & 1.5 & & 110216 & 7.875 \\
\hline
\end{tabular}

No significant reduction in stress is seen by using model 3 with $r_{1} / b=0.05$ and 0.1 . However when model 3 is coupled with $r_{1} / b=0.2$ and placed at close vicinity of the central hole it reduces stress by as much as $11 \%$ in plate $\mathrm{C}$. These four auxiliary holes combined has an area of $1.3 \%$ of the plate which sufficiently meets our concern of removing minimum material to attain maximum stress reduction. Model 3 with $\mathrm{r}_{1} / \mathrm{b}=0.2$ has stress reduction effect for all distances but the reduction effect eases out as we move away from the central hole. However it should be noted that the auxiliary holes must be placed beyond $X_{1}=0.5 \mathrm{~b}$ and should not be very near the edge of the plate. In Fig. 9 the area under blue and dark blue colour is significantly less than in Fig. 3. This means that due to the presence of auxiliary holes the stresses are better distributed in model 3.

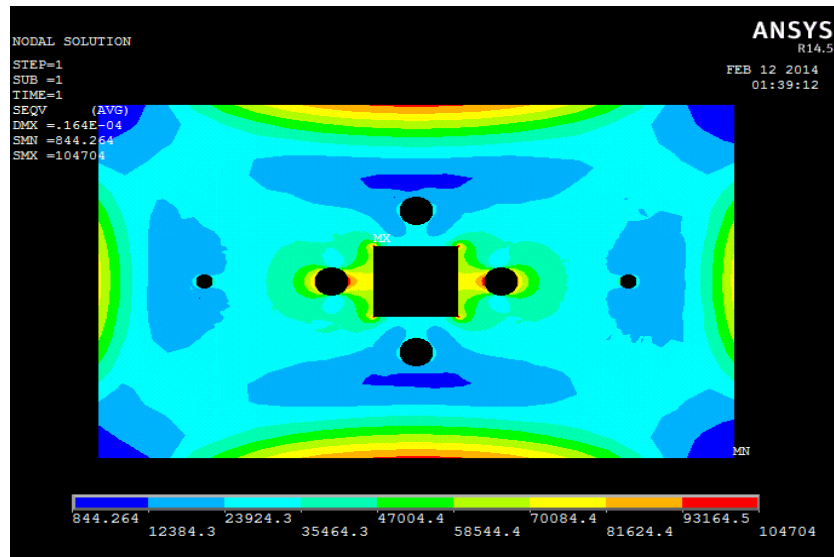

Fig. 11: Stress Contour Plot for Model 4 with $r_{2}=0.1 b, X_{2}=1.5 b$

Further modifications are made to improve the results obtained from model 3. Here simulations are done by taking $r_{1}=0.2$ and $X_{1}=Y_{1}=0.5$ and varying the distance $X_{2}$ and radius $r_{2}$. Two more auxiliary hole at distance $X_{2}$ are drilled. It is observed that at very small radius i.e. $r_{2} / b=0.05$ and 0.1 these 2 nd set of auxiliary hole aid in reducing stress to about $13 \%$. Increasing the radius $r_{2}$ has a poor effect on stress reduction as seen in case of $r_{2} / b=0.2$. When a comparatively larger 2nd pair of auxiliary hole is placed near the 1st set of auxiliary hole the space between this two sets of auxiliary holes (in this case denoted as $\mathrm{X}_{2}$ ) will become a region of high stress concentration. Hence it should be kept in mind that if the plate dimensions are small, the use of 2 nd set of auxiliary holes may not yield good results. 


\section{Conclusion}

Any abrupt change in dimensions gives rise to high stresses around the discontinuity and change in stress flow lines is seen. Through gradual change in the structure reduction in these accumulated stresses is seen. In case of plates with central cutouts this can be achieved by proposed scheme of drilling auxiliary holes around the central hole periphery. The distance should not be less than 0.5 times the dimension of the cutout. In general model 2 and model 3 seems to work better at cutout proximity of about 0.5 times the central cutout dimension. The removal of material by inclusion of auxiliary holes to reduce stress is practically more suitable for plate with infinite dimension due to sufficient availability of space and would lower the stress by significant amount. It is observed that symmetric auxiliary holes around the central hole have better stress reduction. Also if sufficient space is available, a second smaller set of auxiliary holes will further augment the stress reduction process. By using model 4 all the auxiliary holes together occupied an area of only $1.5 \%$ of the plate area for a reduction of stress of more than $12.5 \%$.

\section{Acknowledgements}

The author is thankful to Dr. Salil Haldar, Professor and Head, Dept. of Aerospace Engineering \& Applied Mechanics, IIEST Shibpur for his valuable insights on the paper.

\section{References}

[1] Motok, M. D. "Stress concentration on the contour of a plate opening of an arbitrary corner radius of curvature." Marine structures 10.1 (1997): 1-12. http://dx.doi.org/10.1016/S0951-8339(96)00012-3

[2] Heywood, Roland Bryon. Designing by photo elasticity. Chapman \& Hall, 1952.

[3] Durelli, A. J., and K. Rajaiah. "Optimum hole shapes in finite plates under uniaxial load." Journal of Applied Mechanics 46.3 (1979): 691695. http://dx.doi.org/10.1115/1.3424628

[4] Meguid, S. A. "Finite element analysis of defence hole systems for the reduction of stress concentration in a uniaxially-loaded plate with two coaxial holes." Engineering fracture mechanics 25.4 (1986): 403-413. http://dx.doi.org/10.1016/0013-7944(86)90254-7

[5] Giare, G. S., and R. Shabahang. "The reduction of stress concentration around the hole in an isotropic plate using composite materials." Engineering fracture mechanics 32.5 (1989): 757-766. http://dx.doi.org/10.1016/0013-7944(89)90172-0

[6] K. Kalita and A. K. Banerjee, Static Analysis of Isotropic \& Orthotropic Plates with Central Cutout under Transverse Loading, Proc. of NCRAME (2013), 57-63.

[7] Kalita, Kanak, Abhik Kumar Banerjee, and Salil Haldar. "An Analysis to Mitigate Induced Stresses in Orthotropic Plates with Central Square Cut-out." International Journal of Engineering 6.3 (2013): 379-386.

[8] Kalita, Kanak and Salil Haldar. "Stress concentration minimization of 2D simply supported perforated steel plate", International Journal on Theoretical and Applied Research in Mechanical Engineering 3.1 (2014): 50-55.

[9] Timoshenko, S. P., and J. N. Goodier. "Theory of elasticity, 1987."

[10] Dally, James W., and William F. Riley. "Experimental stress analysis, 1991."McGraw and Hill, New York.

[11] Savin, Guriǐ Nikolaevich. Stress concentration around holes. Vol. 1. Pergamon Press, 1961. 